\title{
Laser-generated plasma plume expansion: Combined continuous-microscopic modeling
}

\author{
Tatiana E. Itina,* Jörg Hermann, Philippe Delaporte, and Marc Sentis \\ Laboratoire Lasers, Plasmas et Procedés Photoniques, LP3-FRE 2165 CNRS, Faculté des Sciences de Luminy, Case 917, \\ 13288 Marseille, Cedex 9, France \\ (Received 15 January 2002; published 12 December 2002)
}

\begin{abstract}
The physical phenomena involved in the interaction of a laser-generated plasma plume with a background gas are studied numerically. A three-dimensional combined model is developed to describe the plasma plume formation and its expansion in vacuum or into a background gas. The proposed approach takes advantages of both continuous and microscopic descriptions. The simulation technique is suitable for the simulation of high-rate laser ablation for a wide range of background pressure. The model takes into account the mass diffusion and the energy exchange between the ablated and background species, as well as the collective motion of the ablated species and the background-gas particles. The developed approach is used to investigate the influence of the background gas on the expansion dynamics of the plume obtained during the laser ablation of aluminum. At moderate pressures, both plume and gas compressions are weak and the process is mainly governed by the diffusive mixing. At higher pressures, the interaction is determined by the plume-gas pressure interplay, the plume front is strongly compressed, and its center exhibits oscillations. In this case, the snowplough effect takes place, leading to the formation of a compressed gas layer in front of the plume. The background pressure needed for the beginning of the snowplough effect is determined from the plume and gas density profiles obtained at various pressures. Simulation results are compared with experimentally measured density distributions. It is shown that the calculations suggest localized formation of molecules during reactive laser ablation.
\end{abstract}

DOI: 10.1103/PhysRevE.66.066406

PACS number(s): 52.38.Mf, 02.60.Cb

\section{INTRODUCTION}

During the last decade, pulsed laser ablation has found a number of applications in such fields as laser treatment of surfaces, pulsed laser deposition (PLD) of thin films, mass spectrometry, and nanotechnology [1-3]. The further development of these applications requires a better understanding of the mechanisms of laser-solid interaction, of the formation of a plasma plume, and of its expansion under vacuum or in the presence of a background gas. Numerous experiments have been performed demonstrating the complexity of the physical processes involved in the laser ablation phenomenon [4-11]. To explain the experimentally observed processes, a number of analytical and numerical models have been proposed [12-33]. These models provided rich scientific information about the properties of the ablated particles, plasma content, and the dynamics of the laser plume expansion.

Two extreme cases of either very low or relatively high background pressure are considered in most theoretical studies of laser plume expansion. The plume expansion into vacuum can be described as self-similar adiabatic [12,34]. At a pressure of some pascals, the expansion is governed by diffusion, ablated particles are thermalized in collisions with the background gas, and collective effects of the plumebackground-gas interaction can be disregarded [10,21,22]. In this case, the plume expansion process can be divided into two stages: (i) the plume formation and (ii) the propagation of the ablated species through a background gas. A combi-

\footnotetext{
*Corresponding author. FAX: (+33) 4918292 89. Email address: itina@1p3.univ-mrs.fr
}

nation of two Monte Carlo techniques (direct Monte Carlo and random trajectories) was shown to be effective for the calculation of the particle transport up to a distance of several centimeters from the irradiated target [22]. In another extreme case of ambient pressure as high as some hundreds of pascals, shock waves were shown to be produced during the plume expansion into background gases [34,35]. The formation and propagation of these shock waves are found to be satisfactorily described by the analytical "blast-wave," or "point explosion" model [35] that disregards the energy dissipation and particle diffusion. In addition, both one- and two-dimensional numerical models were proposed to describe the dynamics of shock wave formation and propagation for plasma expansion in high-pressure background gases $[27,28,30]$.

Only a few studies were undertaken to describe the plume expansion at intermediate background pressures (between 10 and $100 \mathrm{~Pa}$ ). This case is the most complicated one because of the importance of the collective effects leading to the compression of both the plume and the gas, as well as to the plume-gas diffusion. Adiabatic models cannot be applied, and the plume-gas mass and energy exchange should be taken into account. To describe the plume propagation under such conditions, a phenomenological drag model [36] was proposed, which uses fitting parameters. In another model, collision effects were introduced into a system of gasdynamical equations, thus allowing the description of the plume splitting and of its edge heating [32]. An analytical model that considers plume-gas energy exchange was, furthermore, proposed for spherical laser plume expansion in the intermediate pressure regime [29]. This model explained the interaction between the plume and the background gas in terms of the formation of internal and external shock waves, but disregarded the plume-gas mixing. For three-dimensional 
expansion, a system of Navier-Stokes gas-dynamical equations together with Fourier's law for the heat-conductive flux and Fick's equation for diffusion fluxes [33] was solved numerically. The numerical solution provided a wealth of information about the first 1-2 $\mu \mathrm{s}$ of the plasma plume expansion. Gas-dynamical models are, however, invalid when the velocity distribution of the ablated particles deviates from the Maxwellian distribution. As a result, they cannot correctly be applied for the regions of strong density gradients. In addition, diffusive processes are not satisfactorily described by the continuous gas dynamics [33], and the shock front thickness is frequently underestimated. The mixing of the ablated species with the background gas cannot be rigorously studied with these models. The latter process, however, always takes place at the later stage of the plume expansion and strongly influences the properties of the ablated species. In addition, an effective mixing between the ablated and background particles is indispensable in the reactive laser ablation, because it provides the conditions required for the formation of the reactive products.

The difficulties of the gas-dynamical description of the diffusive processes are avoided in direct simulation Monte Carlo (DSMC) method [23,25,37-39]. In this method, physical space is subdivided into a system of cells, where the cell size is determined by the mean free path of the particles. A representative number of molecular collisions should be calculated for each cell. As a result, this method is computationally effective if the Knudsen number $(\mathrm{Kn}=l / L$, where $l$ is the mean free path and $L$ is a characteristic length) is not very small. During the laser plume expansion, however, a very strong variation of the Knudsen number (typically, from $\sim 10^{-5}$ to 1 ) takes place. When the DSMC is used to simulate the plume expansion process, most of the computer resources are used to calculate only the initial stage of the expansion, which lasts for several laser pulse durations. In the presence of a background gas, additional collisions between the plume and the background-gas molecules should be considered. Furthermore, the method does not describe ion formation and recombination that are particularly significant at the beginning of the plume expansion. As a result, the DSMC becomes computationally expensive (in terms of computer memory and calculation time) in the case of highrate laser ablation in the presence of a background gas with pressure more than $\sim 10 \mathrm{~Pa}[40]$.

To our knowledge, no universal model has been proposed to effectively study the different regimes of the plume expansion. We believe that a combination of different approaches can decrease a number of model limitations. Therefore, in the present paper, we propose a hybrid method, which combines both continuous and microscopic descriptions of laserinduced plasma expansion. The developed approach is applicable for plume expansions in different background pressures, ranging from vacuum up to the regime of strong shock waves. The method is particularly useful for studying the transitional background pressure regime. It takes advantage both of the gas-dynamical description of the plume at the early stage, and of the DSMC simulation at the late stage of the plume expansion, avoiding the above-described challenging issues of these techniques. As a result, the expansion of a high-density plume in the presence of a background gas can be tackled during a time as long as $5-10 \mu$ s at ambient gas pressures from 0 to some hundreds of pascals. Using the capacities of the proposed model, different regimes of the plume expansion are determined as a function of the background pressure. Such effects as plume deceleration and snowplowing, plume-gas mixing, backscattering of the ejected particles, and their interaction with the irradiated surface are investigated.

To validate the model, and to investigate the plume expansion under different background pressures, we use laserinduced fluorescence spectroscopy of the plume species. This technique allows one to measure ground-state number densities of atoms and molecules in plasma plumes [41]. In the present paper, the ablation of metal targets in the presence of oxygen background gas is investigated. Three-dimensional number density mapping of plasma species are performed for different values of the background pressure. Fluorescence probing of the diatomic oxide molecules formed as a result of the interaction of the ablated species with the oxygen background is used to visualize the zones of the strong mixing of metal atoms with oxygen molecules. The obtained experimental results are explained based on the numerical simulation.

\section{NUMERICAL MODELING: COMBINED LARGE-PARTICLE-DSMC METHOD}

\section{A. General considerations}

Previous studies showed that during the early stage of the plume development and expansion, the plume-background gas diffusion is negligible, and energy exchange can be disregarded $[33,30]$. Because diffusion and dissipation present a challenge in the continuum modeling approach, the key idea of the proposed simulation method is to combine the continuum gas-dynamical modeling for the initial stage of the plume expansion with the direct Monte Carlo simulation of the ablated flux into a background gas at the later stage.

The developed model is three dimensional and uses axial symmetry. We assume that a laser beam with radius $R_{0}$ and pulse duration $\tau$ interacts with a plane target $(z \leqslant 0)$. The half space $z>0$ is filled by a background gas (pressure $P_{b}$, density $\rho_{b}$ ). Under laser irradiation, the target absorbs a part of the laser energy, the target material is heated and ablated forming a laser plume near the target surface.

\section{B. First stage of plasma plume expansion: The method of large particles}

At the first stage, we consider the plasma plume as a nonviscous and non-heat-conductive vapor containing atoms, ions, and electrons. In the plume, the temperature of electrons, $T_{e}$, deviates from that of ions and neutrals, $T_{a}$, because of the slow rate of energy transfer between electrons and heavy particles. Based on these considerations, we adopt an approach of one-fluid two-temperature gas dynamics to describe the movement of the laser plume. In this case, the system of gas-dynamical equations can be written in the divergent form as follows (the plume expands along the $Z$ axis): 


$$
\begin{gathered}
\frac{\partial \rho}{\partial t}+\operatorname{div}(\rho \vec{W})=0 \\
\frac{\partial \rho U}{\partial t}+\operatorname{div}(\rho U \vec{W})+\frac{\partial P}{\partial z}=0, \\
\frac{\partial \rho V}{\partial t}+\operatorname{div}(\rho V \vec{W})+\frac{\partial P}{\partial r}=0, \\
\frac{\partial \rho E_{a}}{\partial t}+\operatorname{div}\left(\rho E_{a} \vec{W}\right)+\operatorname{div}\left(P_{a} \vec{W}\right)=Q_{\mathrm{ei}}+Q_{\mathrm{ea}}, \\
\frac{\partial \rho E}{\partial t}+\operatorname{div}(\rho E \vec{W})+\operatorname{div}(P \vec{W})=-F, \\
E=\varepsilon_{a}+\varepsilon_{e}+|\vec{W}|^{2} / 2, \quad E_{a}=\varepsilon_{a}|\vec{W}|^{2} / 2, \\
P=P_{a}+P_{e}, \quad P_{a}=(\gamma-1) \rho \varepsilon_{a}, \quad P_{e}=(\gamma-1) \rho_{e} \varepsilon_{e},
\end{gathered}
$$

where $\rho$ is the density, $U$ and $V$ are the $Z$ and $R$ components of the velocity vector $\vec{W}, \gamma$ is the specific heat ratio, $\varepsilon_{a}$ is the thermal energy of ions and neutrals, $\varepsilon_{e}$ is the thermal energy of the electron gas, and $P_{a}$ and $P_{e}$ are the partial pressures of the heavy (ions and neutrals) and light (electrons) gases. In Eqs. (4) and (5), $Q_{\text {ei }}$ and $Q_{\text {ea }}$ describe the energy exchange between the electron gas and the gas composed of ions and neutrals, and $F$ is the energy lost by electrons for ionization. In Eqs. (1)-(7), a local thermodynamic equilibrium is assumed, and ideal-gas equations of state are used.

To solve numerically the system (1)-(7) we use the method of large particles (LP) adapted to the case of lasersolid interaction $[30,42,43]$. The advantages of this method are the uncoupling of physical processes and the combination of the Euler and Lagrange approaches, which gives the possibility of following initial plume expansion into a background gas in an affordable computer time. In the present model, for simplicity, we consider only singly charged ions, and use the equilibrium ionization-recombination kinetics. Photoionization and photorecombination processes, as well as any kind of laser-vapor interaction are neglected.

The heat obtained by ions and atoms in elastic collisions with electrons is

$$
Q_{\mathrm{ei}}+Q_{\mathrm{ea}}=\frac{2 m_{e}}{m} \frac{3}{2} k\left(T_{e}-T_{a}\right) n_{e}\left(\nu_{\mathrm{ei}}+\nu_{\mathrm{ea}}\right)
$$

where $m_{e}$ is the electron mass, $n_{e}$ is the electron number density, and $\nu_{\text {ei }}$ and $\nu_{\text {ea }}$ are the mean frequencies of electronion (ei) and electron-atom (ea) collisions, respectively [34]. The collision frequencies are

$$
\begin{gathered}
\nu_{\mathrm{ei}}=\frac{4(2 \pi)^{1 / 2} n_{i} e^{4} \Lambda_{C}}{3 m_{e}^{1 / 2}\left(k T_{e}\right)^{3 / 2}}, \\
\nu_{\mathrm{ea}}=\frac{\bar{c} \sum n_{a} n_{e}}{2},
\end{gathered}
$$

where $\Lambda_{C}$ is the Coulomb logarithm, $\bar{c}$ is the average relative velocity, and $\Sigma$ is the electron-atom collision cross section. The energy lost by electrons for ionization,

$$
F=\frac{2 n_{e} n_{a} \varepsilon_{i} k_{\mathrm{ei}}}{3}
$$

where

$$
k_{\mathrm{ei}}=2 \frac{\left(2 \pi m_{e} k T_{e}\right)^{3 / 2}}{h^{3} n_{e}} k_{\mathrm{rec}} \exp \left(-\frac{\varepsilon_{i}}{T_{e}}\right),
$$

is the $e$-impact ionization rate constant and

$$
k_{\mathrm{rec}}=\frac{4 \pi(2 \pi)^{1 / 2}}{9} \frac{e^{10}}{m_{e}^{1 / 2}\left(k T_{e}\right)^{9 / 2}} n_{e}
$$

is the three-body recombination constant $[33,34]$.

Initial boundary conditions at the surface $z=0$ are calculated as a heat source resulting from the absorption of the laser radiation corresponding to a laser pulse with a Gaussian temporal shape and pulse duration $\tau$. The thermal evaporation model is used, so that the vapor pressure at the surface is obtained from the Clasius-Clapeyron equation [15]. The temperature distribution along the laser-irradiated area is assumed to be uniform. The electron gas temperature at the beginning is set to be higher than that of heavy atoms, because electrons absorb laser radiation. The initial flow parameters at $z=0$ and $r<R_{0}$ are calculated using the jump conditions at the Knudsen layer boundary [44,45]. The flow velocity at the Knudsen layer boundary $U_{K}$ is assumed to be equal to the velocity of sound $a_{K}$, so that the Mach number $M=1$ at $z=0$ [45]. For one-atomic vapor $\left(\gamma=\frac{5}{3}\right)$, the Knudsen layer parameters are $T_{K}=0.65 T_{S}, n_{K}=0.62 n_{S}, U_{K}$ $=1.31 T_{S}$, where index $S$ refers to the parameters at the surface, and $K$ to the border of the Knudsen layer.

The large-particle calculations are performed until a certain time $t_{0}$, and then, the motion of the ablated and background species is followed by using a microscopic Monte Carlo method. On one hand, the switch time $t_{0}$ should be long enough for the plume density to diminish by several orders of magnitude with respect to the initial values. As a result of the plume expansion, the characteristic Knudsen number drops down to the values required for the effective application of the DSMC method. Moreover, the $t_{0}$ should be long enough to account for the ionization-recombination processes in the plasma plume. On the other hand, the switch time should be short enough, such that the mass diffusion and the heat exchange between the plume and the background gas are insignificant during $t_{0}$.

\section{Second stage of plume expansion into a background gas: Direct simulation Monte Carlo}

At $t>t_{0}$, the ablated plume expansion into a background gas is calculated using the DSMC method [37]. In this method, gas flows are modeled by a representative ensemble of atoms or molecules (typically from $10^{3}$ to $10^{6}$ ). The DSMC procedure uses the uncoupling of the molecular motion and the intermolecular collisions. The method has the 
same limitations as kinetic theory of gases, which includes the Boltzmann equation. The principal limitations are the assumption of molecular chaos and requirement of a dilute gas. The details of the method can be found in Ref. [37] and below we only briefly describe the procedure.

The simulated region is divided into a network of axial cells, each cell containing four subcells. The subcell dimensions are smaller than the local mean free path, and increase with the distance from the target and from the plume axis. The time is incremented by discrete steps, $\Delta t$. Because the laser plume expands and its density drops rapidly with time, the size of the simulated region, the cell dimensions, and the time step are increased six times during the simulation. Simulation particles are introduced randomly based on the spatial distributions of ablated particle and gas number densities obtained from the large-particle simulation. The velocities of the ablated atoms and ambient gas molecules are sampled from the local Maxwellian distribution using temperature and flow velocity distributions obtained from the LP simulation. Weighing factors are used to decrease the statistical error in cells that are close to the plume axis.

The intermolecular collisions are calculated using a notime counter (NTC) schema [37]. In this method, the following number of collision pairs:

$$
N_{P}=(1 / 2) N_{S} \bar{N}_{S} Q\left(\sigma_{T} c_{r}\right)_{\max } \Delta t / V_{C}
$$

are selected from each cell during one time step, where $N_{S}$ and $\bar{N}_{S}$ are, respectively, the instantaneous and the average number of simulated molecules in the cell, $c_{r}$ is the relative speed of the colliding particles, $\sigma_{T}$ is the collision cross section, $\left(\sigma_{T} c_{r}\right)_{\max }$ stands for the maximum product in the cell, and $V_{C}$ is the cell volume. The factor $Q$ defines the number of real particles represented by each simulated molecule, so that collision frequencies in the simulation are identical to the real ones. Each collision is computed with the probability

$$
P=\frac{\sigma_{T} c_{r}}{\left(\sigma_{T} c_{r}\right)_{\max }}
$$

The procedure is species independent and the NTC method is best applied to all species as a single group.

For computing the postcollision velocities, a variable hard sphere model (VHS) is used [37]. This model realistically describes the temperature exponent of the coefficient of viscosity. Unlike the well-known hard sphere model, the diameter of VHS molecules depends on the relative velocity

$$
d=d_{\text {ref }}\left(c_{r \text { ref }} / c_{r}\right)^{v},
$$

where the subscript ref denotes reference values, and $v$ is a parameter determined by the viscosity temperature power law. In the inelastic collisions with diatomic molecules, the internal (rotational) modes of the molecules are described by a Larsen-Borgnakke model [37].

Microscopic boundary conditions are specified by the behavior of the individual particles, rather than in terms of the distribution function. The interaction of the backscattered atoms with the target follows the Cercignani-Lampis-Lord (CLL) model [37].

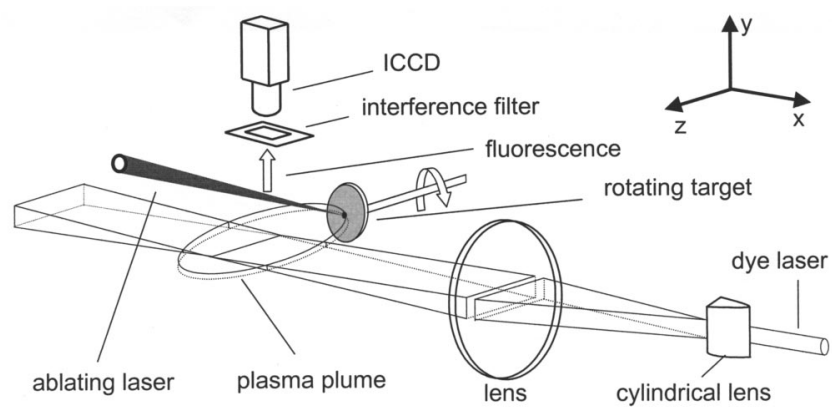

FIG. 1. Geometry of laser-induced fluorescence measurements.

\section{EXPERIMENTAL PROCEDURE}

A frequency quadrupled Nd:YAG (yttrium aluminum garnet) $(266 \mathrm{~nm}$ ) laser (Quantel, Brilliant) with pulse duration $\tau=5 \mathrm{~ns}$ and laser energy of $15 \mathrm{~mJ}$ was used for target ablation in the laser-induced fluorescence experiments (Fig. 1). The pulse repetition rate was $0.3 \mathrm{~Hz}$, and the laser beam was focused onto a spot with radius $R_{0}=0.15 \mathrm{~mm}$. Metal targets ( $\mathrm{Al}$ and $\mathrm{Ti})$ were placed in a vacuum chamber, which was evacuated to a residual pressure of about $0.1 \mathrm{~Pa}$. During laser irradiation, the pumping rate was reduced and a background gas $\left(\mathrm{O}_{2}\right)$ was introduced. The background pressure was varied from 1 to $70 \mathrm{~Pa}$. The target was rotated to avoid drilling. The probe laser beam was delivered by a $\mathrm{XeCl}$ excimer laser pumping a tunable dye laser. By using the Coumarin 307 dye laser, the system generated pulses of $20 \mathrm{~ns}$ duration and 10 $\mathrm{mJ}$ energy at $500 \mathrm{~nm}$. A beam expander was used to transform the probe laser beam in a thin plane section of 0.2 $\times 40 \mathrm{~mm}^{2}$ dimension as shown in Fig. 1. The fluorescence signal was captured using a focusing lens and a fast intensified charge coupled device (ICCD) with a 50-ns gate. The synchronization between ablating laser, probe laser, and ICCD was ensured by using the $Q$-switch trigger output of the Nd:YAG laser. The gate was set to a time delay corresponding to the maximum intensity of fluorescence emission. To enhance the signal to noise ratio, data acquisition was performed by averaging the signal over several ablation events. More details about the experimental procedure can be found elsewhere [41].

\section{SIMULATION RESULTS AND COMPARISON WITH EXPERIMENTS}

\section{A. Simulation parameters and basic assumptions}

The developed combined LP-DMCS method is used for the calculations of the laser ablation of aluminum in the presence of an oxygen background gas. Laser parameters are set to be the similar to these in the experiments. The parameters defining thermal properties of the irradiated target are the same as in Ref. [46]. The ambient gas is assumed to be at standard temperature. Large-particle calculations are performed till $t_{0}=10 \tau=50 \mathrm{~ns}$, and then the DMCS method is used. The calculation parameters used in the second part (DMCS) are presented in Table I [47]. Here, the van der Waals radius is used for aluminum, as a reference diameter. 
TABLE I. Parameters used in the DSMC calculations.

\begin{tabular}{lrr}
\hline \hline & \multicolumn{1}{c}{$\mathrm{Al}$} & \multicolumn{1}{c}{$\mathrm{O}_{2}$} \\
\hline Reference diameter $d_{\text {ref }}(\AA)$ & 4.10 & 3.96 \\
Molecular weight $(\mathrm{amu})$ & 26.98 & 32.00 \\
Viscosity temperature power & 0.80 & 0.77 \\
$\quad$ law constant, $s=0.5+v$ & & \\
\hline \hline
\end{tabular}

In most of the calculation, the accommodation coefficient, which is used for the CLL interaction with the target, is set to be 0.5 . In addition, several calculations are also performed with diffuse reflection of the backscattered particles from the surface. In the DSMC part, the number of simulated particles is increased from $6 \times 10^{4}$ at the beginning to about $8 \times 10^{6}$ at $2.5 \mu$ s. About 200 runs are performed in each DSMC calculation, and the results are averaged afterwards to obtain a satisfactory precision. By using the LP-DSMC method, the plume dynamics is followed till $6.3 \mu$ s under ambient pressure from 0.07 to $70 \mathrm{~Pa}$.

For simplicity, chemical reactions are not included in the present model. In the presence of oxygen, chemical processes take place both on the metal surface and during the plume expansion. The processes in the laser plume include the dissociation of the molecules, as well as the oxidation of aluminum and oxygen recombination. The heat released and absorbed during the reactions influences the dynamics of the laser plume expansion [23]. For example, chemical reactions were shown to broaden the angular distributions of the ab-

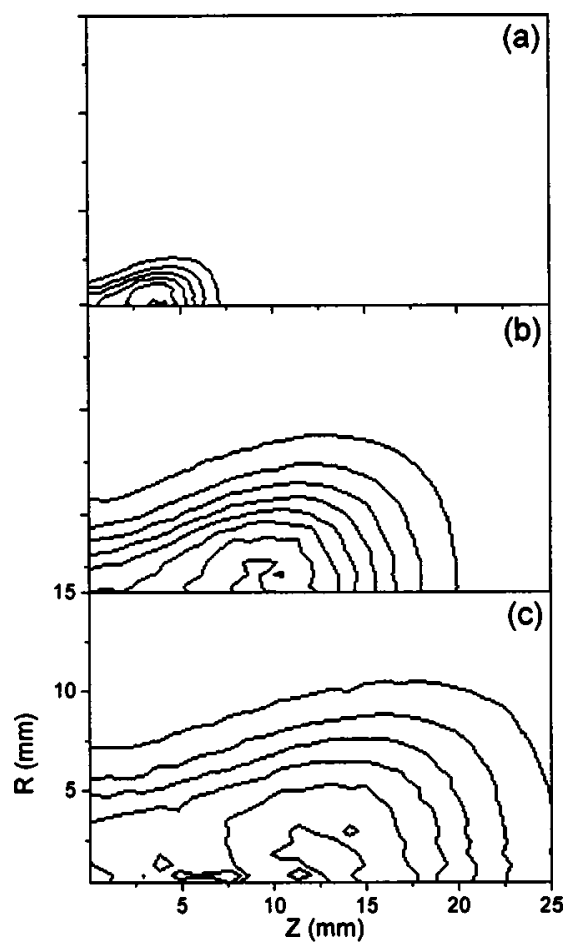

FIG. 2. Contour maps of the calculated spatial distribution of the plume number density as a function of time obtained in calculation with oxygen pressure $P_{b}=0.7 \mathrm{~Pa}$. (a) $t=0.6 \mu \mathrm{s}$, (b) $t=1.5 \mu \mathrm{s}$, and (c) $t=2.7 \mu \mathrm{s}$.

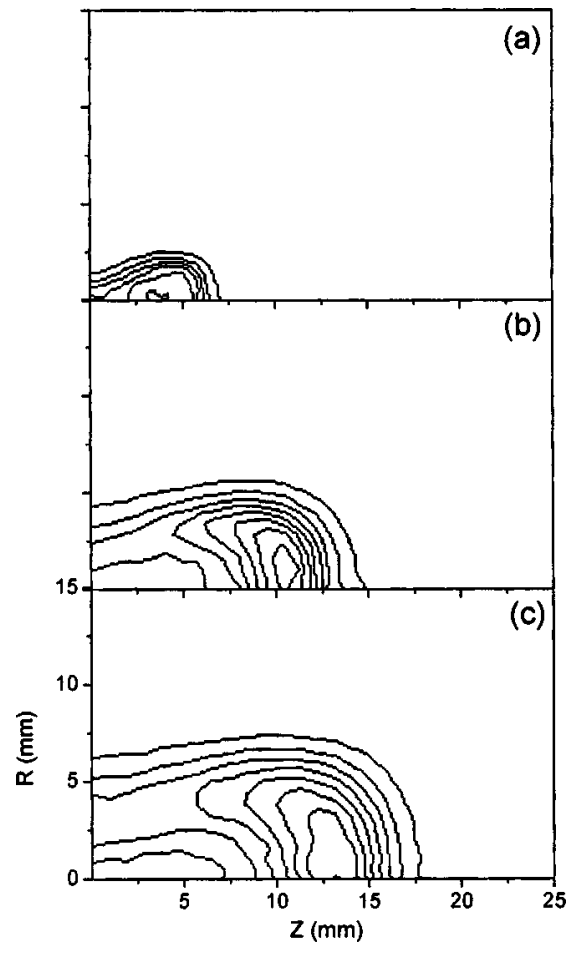

FIG. 3. The same as in Fig. 2, but for $P_{b}=13 \mathrm{~Pa}$.

lated species. These processes also lead to the increase in the plume temperature, if exothermic processes (i.e., the recombination reaction) prevail during laser plume expansion. As a result, the plume energy and velocity can differ from the calculated values. Despite the limitations of the present model, the calculations describe the dynamics of the laser plume expansion and plume-gas mixing because many plume-gas interaction features are not strongly affected by the chemical processes [48].

\section{B. Plume interaction with background gas: Three pressure regimes}

Figures 2-4 visualize the dynamics of the plume expansion in the presence of a background gas. Three oxygen pressure values, $P=0.7,13$, and $70 \mathrm{~Pa}$, are picked up to show three typical regimes of the plume-gas interaction. These regimes can be also observed in Fig. 5, which shows the movement of the plume edge and center, as well as the position of the background density peak and minimum. These figures demonstrate the dynamics of the plume-gas interaction and mixing as a function of the background pressure.

(i) At low pressures (Fig. 2), the mean free path of ablated particles in the ambient gas, $l_{a b}$, is on the order of several centimeters. As a result, a strong expansion of the plume is observed, and no compressed layer is formed, neither in the plume, nor in the background gas. The leading edge of the plume moves away from the target with a practically constant velocity $\left(\sim 1.3 \times 10^{4} \mathrm{~m} / \mathrm{s}\right.$ in our case $)$, whereas the velocity of the plume center is almost one order of the magnitude smaller $\left(\sim 1.7 \times 10^{3} \mathrm{~m} / \mathrm{s}\right)$ [Fig. $\left.5(\mathrm{a})\right]$. The plume density drops down rapidly, so that the background gas can easily fill up the region previously occupied by the plume. 


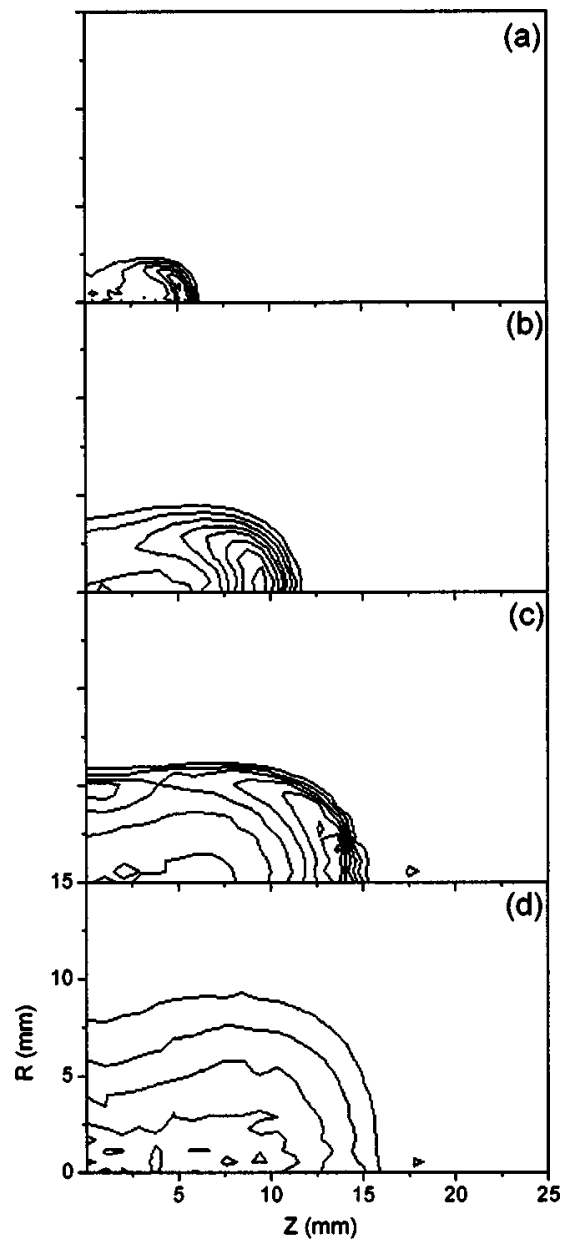

FIG. 4. The same as in Fig. 2, but for $P_{b}=70 \mathrm{~Pa}$. The distributions are obtained at (a) $t=0.6 \mu \mathrm{s}$, (b) $t=1.5 \mu \mathrm{s}$, (c) $t=2.7 \mu \mathrm{s}$, and (d) $t=6.3 \mu$ s from the beginning of the laser pulse.

(ii) At intermediate pressures (Fig. 3), the plume expansion is similar to that in the first case at the beginning (until $t \sim 1 \mu \mathrm{s}$ in our case), and then the plume starts being decelerated. The deceleration begins when plume dimensions become comparable with $l_{a b}\left(l_{a b} \sim 5 \mathrm{~mm}\right.$ at $\left.P_{b}=13 \mathrm{~Pa}\right)$. If the mean free path of the ablated atoms in the plume is smaller than the plume dimensions, ablated atoms interact with each other, and collective effects start playing a role. The plume edge decelerates and stops when the plume dimensions become comparable with the mean free path $(t \sim 2.5 \mu \mathrm{s})$ [Fig. 5(b)]. The plume center is decelerated at about the same time. The gas density peak rises at the beginning of the expansion and then decreases, continuously propagating outward from the target [Fig. 5(d)]. Later (at $t>2.7 \mu \mathrm{s}$ ), the plume expansion is mainly governed by a strong mutual diffusion of the species.

(iii) When pressure is further increased (Fig. 4), the plume-background interaction leads to the so-called snowplough effect. The ablated material pushes the ambient gas away from the target as long as the pressure in the plume overcomes that of the background gas. As a result, both the ablated material and the ambient gas are compressed. The plume compression and deceleration can be seen already at

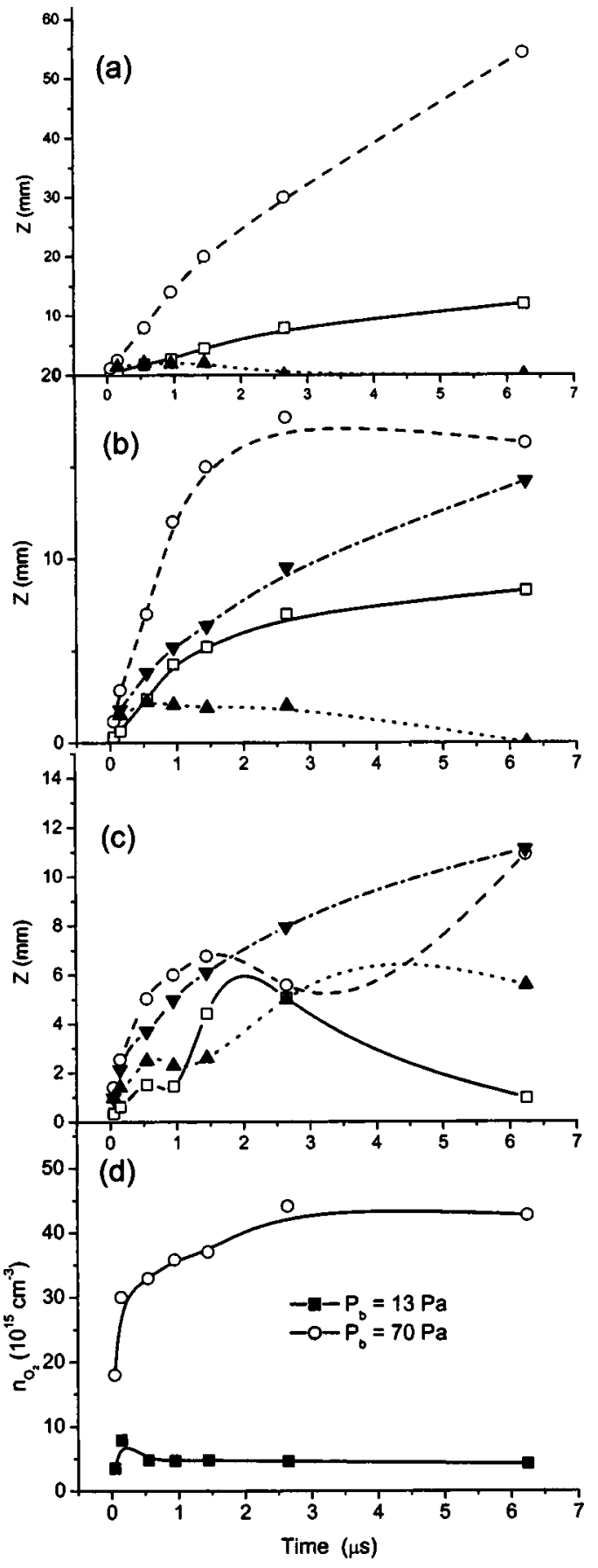

FIG. 5. (a)-(c) Time evolution of the $Z$ position of the plume center $(\square)$, of the plume edge $(\circ)$, of the gas edge $(\boldsymbol{\Delta})$, and of the gas peak $(\boldsymbol{\nabla})$. The presented values are calculated near the plume axis. (a) Oxygen pressure $P_{b}=0.7 \mathrm{~Pa}$, (b) $P_{b}=13 \mathrm{~Pa}$, (c) $P_{b}$ $=70 \mathrm{~Pa}$. (d) Time evolution of the gas peak number density for two different oxygen pressures, $P_{b}=13$ and $70 \mathrm{~Pa}$.

$t=0.6 \mu \mathrm{s}$. At a certain time $(t \sim 2.5 \mu \mathrm{s})$, the plume pressure becomes equal to the background pressure, the plume stops, and its energy is dissipated by the interaction of the compressed plume zone with the compressed background gas. As a result of the interaction, the plume pulsates backward and equilibrates [Fig. 4(b)]. The plume center goes back, thus preventing the background gas from filling up the region 


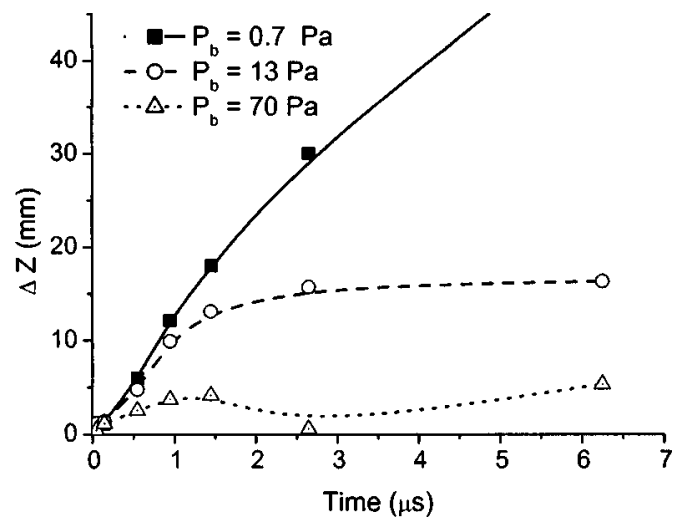

FIG. 6. Effective length of the plume-gas mixing zone, $\Delta Z$ $=Z_{\mathrm{pe}}-Z_{\mathrm{ge}}$, as a function of time for three background pressures.

initially occupied by the plume. After being equilibrated, and if there is still enough energy, the plume will move outward [28]. The plume edge exhibits oscillations [Fig. 5(c)] $[28,25,29]$, whereas the peak of the background density goes away continuously, and its value does not decrease [Fig. $5(\mathrm{~d})]$.

Figure 6 clearly demonstrates how the plume-gas mixing changes with pressure. Here, we follow the time evolution of the effective length of the plume-gas mixing zone, $\Delta Z$ $=Z_{\mathrm{pe}}-Z_{\mathrm{ge}}$, where $Z_{\mathrm{pe}}$ is the position of the plume front, or the $Z$ position where the plume density becomes negligible, and $Z_{\text {ge }}$ is the position of the gas edge. It can be observed that at negligible pressure, the size of the mixing zone rises almost linearly with time. At the intermediate gas pressure, $\Delta Z$ increases rapidly until the beginning of the deceleration $(\sim 3 \mu \mathrm{s})$, and then stabilizes. At higher pressures, oscillations can be observed in the time evolution of $\Delta Z$.

A similar difference in the time evolution of the groundstate atom number density is revealed in the experiments for different background-gas pressures [41]. The experimental results also demonstrate the snowplough effect at an oxygen pressure of $70 \mathrm{~Pa}$. When decreasing the $\mathrm{O}_{2}$ pressure to $13 \mathrm{~Pa}$, the plume expansion mode changes, the process becomes diffusionlike, and no considerable snowplowing is observed at the plume edge.

\section{The influence of the background gas on axial profiles of the plume density and flow velocity}

The calculated axial profiles of the aluminum number density and flow velocity are shown in Figs. 7(a)-7(f). The comparison of the dependencies obtained at different oxygen pressures shows that the background gas affects the plume evolution in two major ways: (i) the plume edge is decelerated so that a density peak appears near it and (ii) the axial flow velocity decreases, and a "plateau" appears at the plume center in its axial distribution. The flow velocity distribution at $P_{b}=13 \mathrm{~Pa}$ demonstrate that most of the plume species move with almost the same flow velocity, and the plume expansion is decreased. The flow velocity, however, rises near the plume "edge" (where density goes to zero) at this pressure. The flow velocity seems to be higher in these regions because only rapid ablated particles propagate till

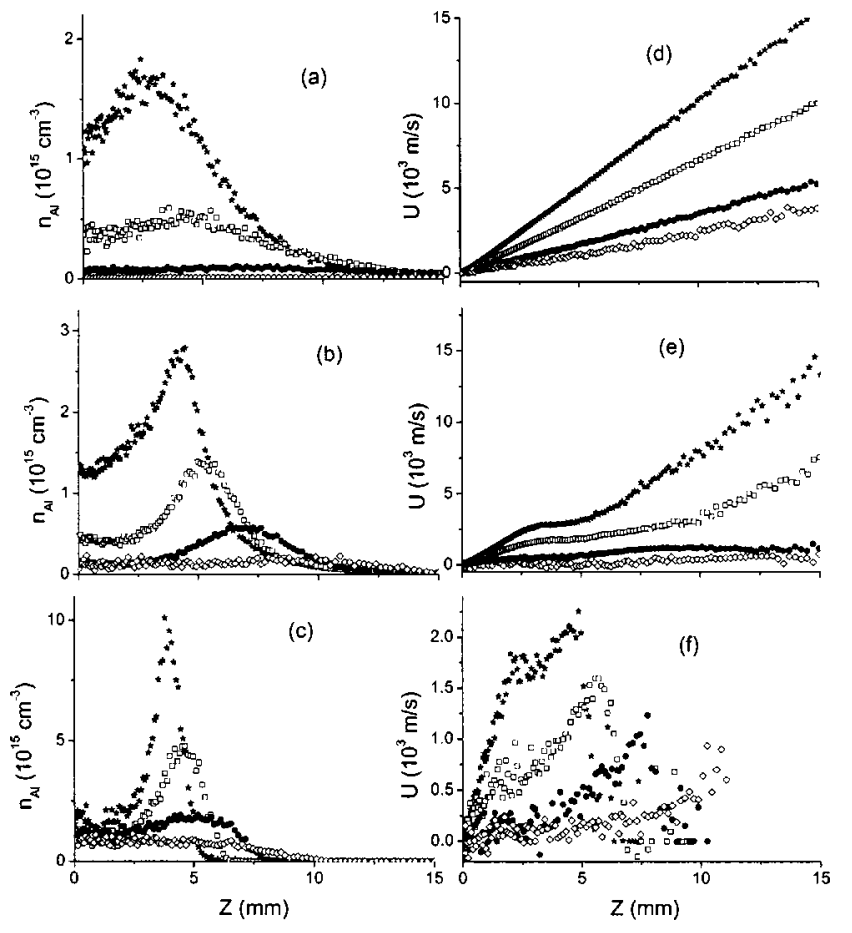

FIG. 7. Calculated axial distributions of the number density (a)(c) and axial flow velocity (d)-(f) at $t=0.6 \mu \mathrm{s}(*), 1.5 \mu \mathrm{s}(\square), 2.7$ $\mu \mathrm{s}(\bullet)$, and $6.3 \mu \mathrm{s}(\diamond)$ from the beginning of the laser pulse. Simulation results presented in (a) and (d) are obtained with $P_{b}$ $=0.7 \mathrm{~Pa}$. Simulation results shown in (b) and (c) are obtained with $P_{b}=13 \mathrm{~Pa}$. (c) and (f) correspond to $P_{b}=70 \mathrm{~Pa}$.

these distances. At $P_{b}=70 \mathrm{~Pa}$, the flow velocity is considerably smaller, and it decreases near the plume edge, indicating the plume "confinement" by the gas. In this case, due to the snowplough effect, even the most rapid ablated particles cannot propagate through the compressed layer.

The calculation results agree with the experimental observations. The measured axial distributions of the number density of ground-state species (Fig. 8) change similarly with time as the calculated dependencies. The density of the ablated species decreases with time, and an abrupt decay can be

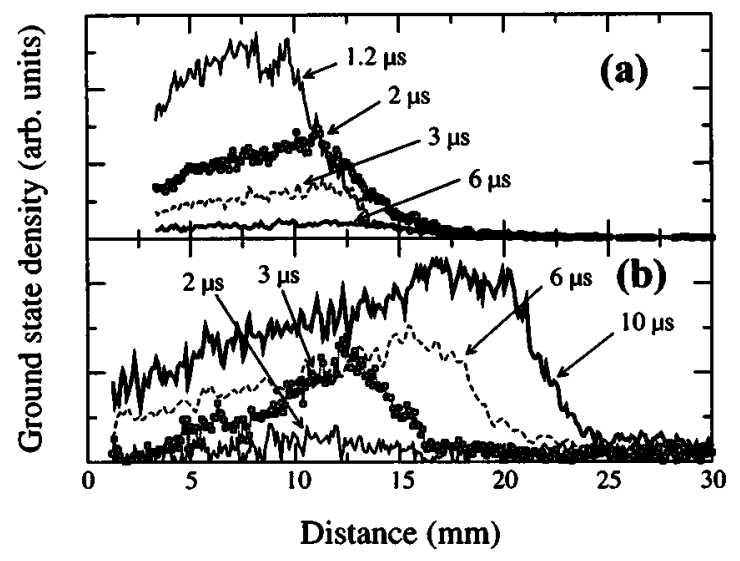

FIG. 8. Experimental axial distributions of ground-state population densities of the plume species as a function of distance from the target at different times. Here $P_{b}=13 \mathrm{~Pa}$, (a) Ti and (b) AlO. 


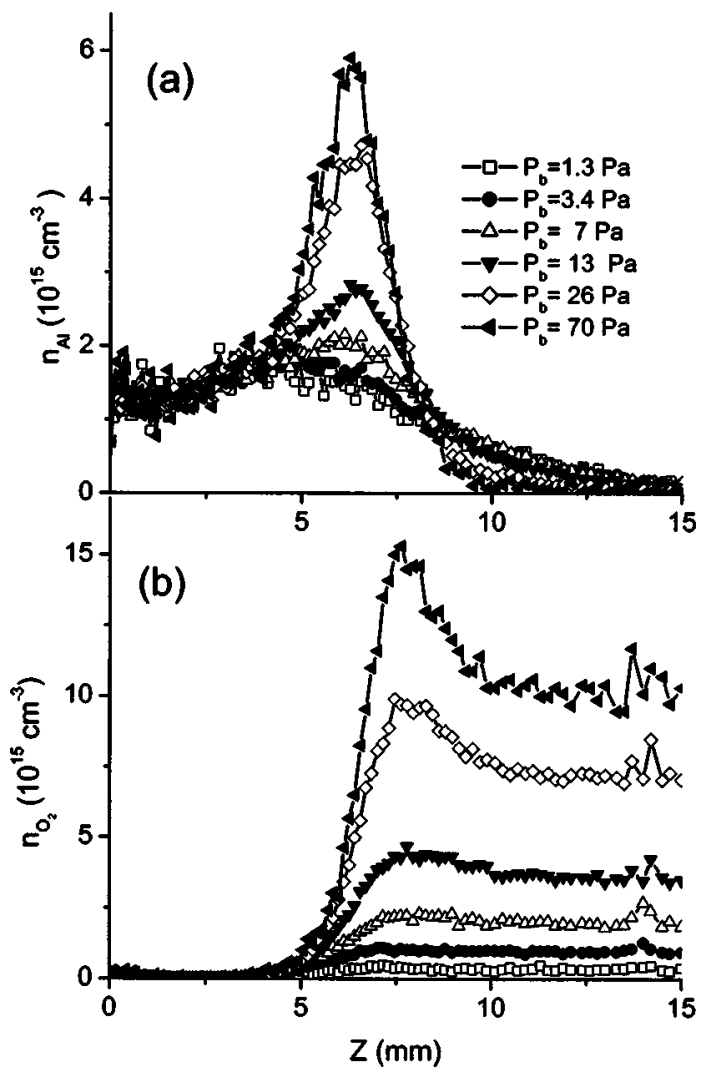

FIG. 9. Calculated axial distributions of the number density of Al species (a) and of oxygen background (b) for different background pressures. The distributions are obtained at $t=1 \mu \mathrm{s}$ from the beginning of the laser pulse.

observed in the distributions near the plume edge. The spatial distribution of the reactively produced $\mathrm{AlO}$ species has its maximum at the region of the plume-gas mixing. Contrarily to the time evolution of the density of the ablated species, the density of the ground-state $\mathrm{AlO}$ molecules increases during the observation time.

The background pressure, which is needed for the beginning of the snowplow, can be determined from the axial density distributions calculated for different background pressures. Figure 9 shows only the beginning of the gas compression $(t=1 \mu \mathrm{s})$, whereas Fig. 10 presents the densities at a later time $(t=2.7 \mu \mathrm{s})$. When $P_{b}=7 \mathrm{~Pa}$, a compression (second maximum) starts appearing in the $n_{\mathrm{Al}}(Z)$ distribution at $1 \mu \mathrm{s}$, indicating the plume splitting [32]. The ambient gas density peak is small at this pressure. With the increase in pressure, more and more ablated material composes the compressed plume region. The peak in the background-gas density becomes well pronounced at $P_{b}$ $=70 \mathrm{~Pa}$, and this pressure can be considered as the one needed for the snowplow effect.

The ambient pressure required for the appearance of the snowplough effect depends on a number of factors, such as molecular weight of the ablated and background species, initial plume velocity, plume density, etc. Evidently, if the ablation rate is not high enough, the plume is too dilute for hydrodynamic effects to play a role, and no snowplow effect takes place. If the plume velocity is small, furthermore,

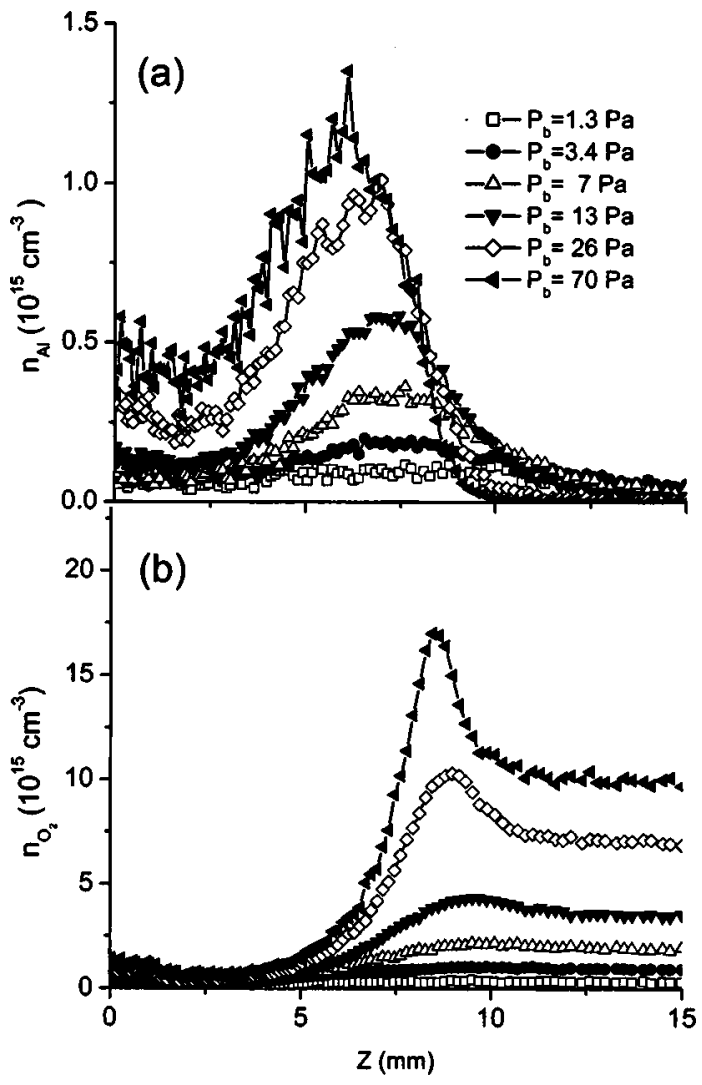

FIG. 10. The same as in Fig. 8, but at $t=2.7 \mu$ s from the beginning of the laser pulse.

shock waves are not formed because the plume expansion should be at least supersonic for their formation.

\section{Plume-ambient gas mixing: Comparison with the experiments}

To investigate the mixing of the plume particles with the background gas in greater detail, we monitor the density of $\mathrm{AlO}$, which is produced during the laser ablation of the aluminum target in the presence of the oxygen background gas. Figure 11 shows the spatial distributions of the AlO groundstate number density $n_{\mathrm{AlO}}^{0}$ experimentally recorded at different pressures and for various time delays. The highest $n_{\mathrm{AlO}}^{0}$ values are observed at the vapor-gas mixing zone, where the interaction between the ablated material and the ambient gas is maximum. The results obtained at the background pressure of $70 \mathrm{~Pa}$ reveal maximum $n_{\mathrm{AlO}}^{0}$ at the plume front, in accordance with the snowplough effect. As we have seen from the calculations at $P=70 \mathrm{~Pa}$, oxygen is deficient at the dense plume center. As a result, no AlO is produced by chemical reactions near the plume center. Strong mixing, which is required for the efficient $\mathrm{AlO}$ production, is realized only in the compressed layer, resulting in the preferential formation of the $\mathrm{AlO}$ in this zone. However, the $n_{\mathrm{AlO}}^{0}$ maps obtained in the experiments at oxygen pressure of $13 \mathrm{~Pa}$ demonstrate a dramatic change in the plume expansion mode. Initially, the maximum $n_{\text {AlO }}^{0}$ values are near the plume edge and at the plume symmetry axis, as at higher pressures. After a certain 


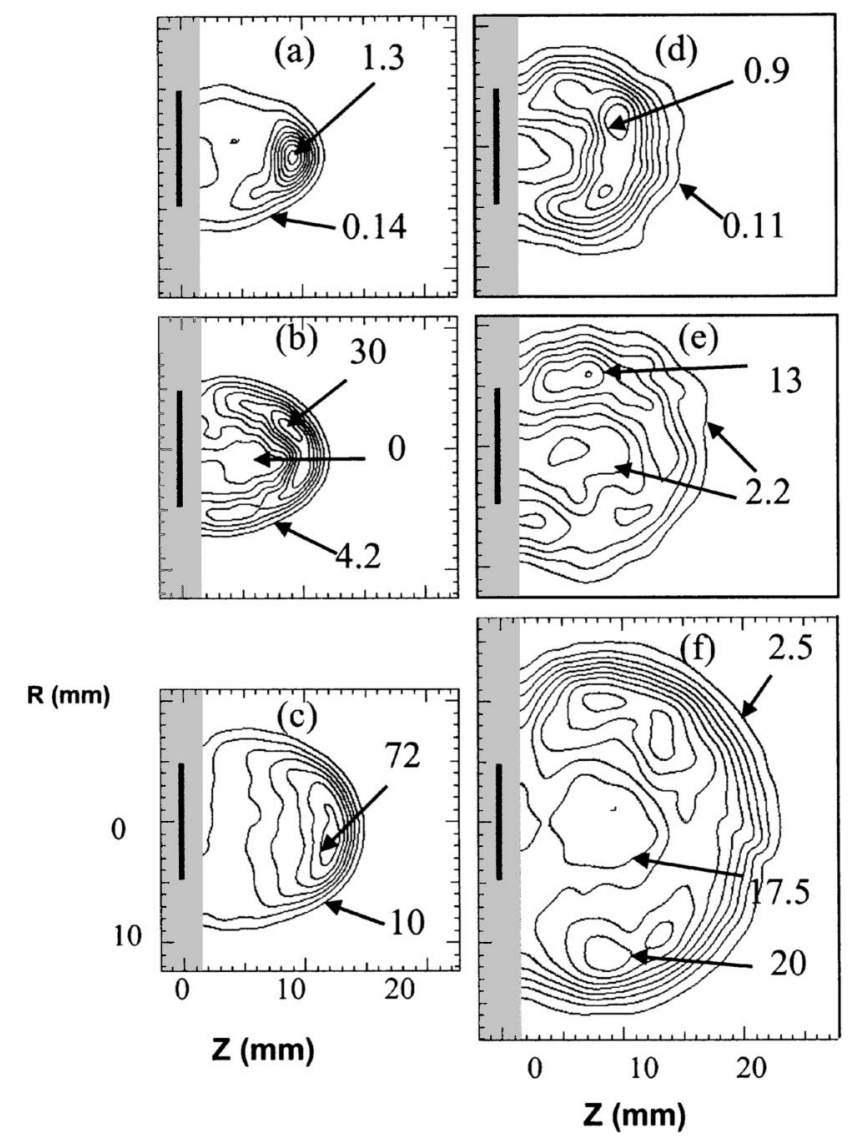

FIG. 11. Contour maps of the experimentally measured AlO ground-state population densities as a function of time for different experimental conditions. Plots (a), (b), and (c) correspond to laser ablation of $\mathrm{Al}$ in $\mathrm{O}_{2}$ at $P_{b}=70 \mathrm{~Pa}$ at $t=2,3$, and $8 \mu \mathrm{s}$, respectively. Plots (d), (e), and (f) show the same distributions at $P_{b}=13 \mathrm{~Pa}$. Numbers indicate maximum and minimum density values in arbitrary units. time, however, the maximum $n_{\mathrm{AlO}}^{0}$ is not detected near the symmetry axis, but at the plume periphery.

The observation of maximum $n_{\mathrm{AlO}}^{0}$ at the plume periphery was previously attributed to the formation of vortices in the gas flow [28]. In fact, an analogy with an underexpanded jet indicated the possibility of such a flow structure as a result of the viscous effects at the plume periphery [28]. In addition, Ref. [25] also reported the observation of the beginning of vortex formation. The simulation results presented in Ref. [25] indicated, however, only the backscattering of ablated and ambient gas particles at the plume periphery near the irradiated target, and no circular motion was observed in the velocity flow field. Nevertheless, vortical flows can be expected to appear due to the formation of a low-pressure zone behind the expanding plume. The presented simulation provides insights into these phenomena.

Typical velocity flow fields obtained from the simulation under conditions close to the experimental ones are presented in Fig. 12. To facilitate the analyses of the flow fields, the plume and background gas density contours are also shown in the same figure. The distribution of the gas velocities demonstrates that background-gas species are entrained in the ablated flow. Furthermore, the velocities of both ablated and background species decrease remarkably at the plume edge. Backward flows of the ablated particles can be revealed at the back sides of the plume at a radial distance of about $5 \mathrm{~mm}$. The regions where the backscattering takes place expand with time. Interestingly, the analyses of the background-gas flow field reveals three different fluxes: (i) one streaming away with the ablated flow, (ii) one scattering in the radial direction and eventually redepositing together with the ablated particles, and (iii) the third one streaming toward the plume axes. The third flux is caused by the presence of a low background density region that has been

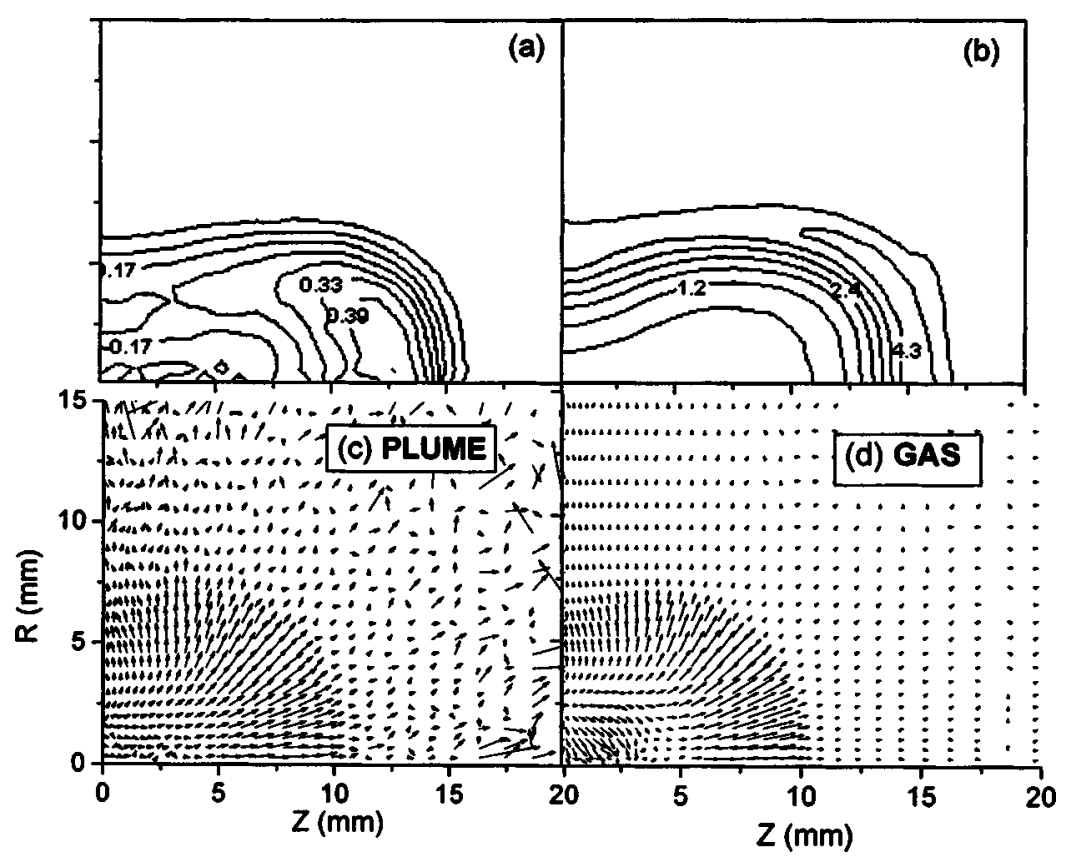

FIG. 12. Spatial distributions of the calculated number density (a), (b) and velocity flow fields (c), (d). The density values are in $10^{20} \mathrm{~m}^{-3}$. Here, the plots (a), (c) correspond to the ablated $\mathrm{Al}$ species, and the plots (b), (c) correspond to the background species. Here, $P_{b}=26 \mathrm{~Pa}$ and $t$ $=2.7 \mu \mathrm{s}$. 


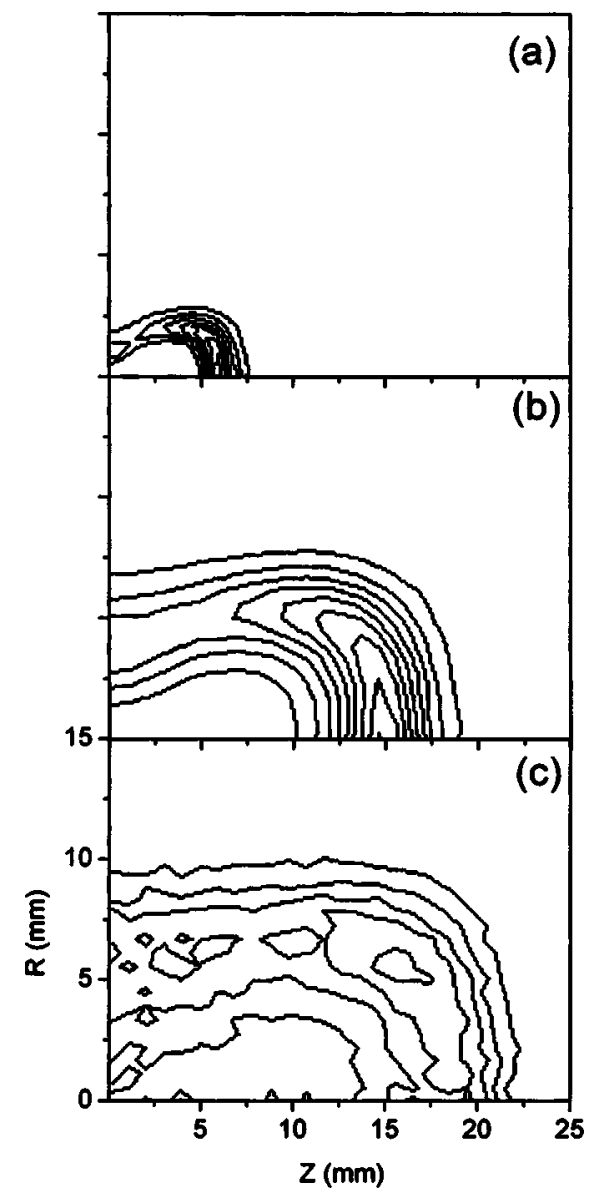

FIG. 13. Calculated spatial distribution of the product of the number densities of $\mathrm{Al}$ and $\mathrm{O}_{2}, n p(r, Z)=n_{\mathrm{Al}_{1}} n_{\mathrm{O}_{2}}$. Here, $P_{b}$ $=13 \mathrm{~Pa}$; (a) $t=0.6 \mu \mathrm{s}$, (b) $t=1.5 \mu \mathrm{s}$, and (c) $t=2.7 \mu \mathrm{s}$.

formed behind the expanding plume [Figs. 12(a) and 12(b)]. In fact, the background gas could not penetrate into this region because it was occupied by the high-density part of the plume. After the plume center has moved away, the background gas molecules fill up this region. Apparently, most of ablated atoms are unaffected by the presence of this region.

No vortices are thus observed in the calculated velocity flow fields. This result can be explained by several factors. First, at the considered oxygen pressures, the mean free path $l_{a b}$ is comparable with the characteristic plume length. The background gas is too rarefied for viscous effects to play a role. In addition, unlike underexpanded jets, the plume expands much stronger in radial directions and its density decreases faster. As a result, the density at the plume periphery does not reach the values needed for the appearance of the viscose effects, such as vortices. Finally, despite the formation of a low-pressure zone behind the plume, it does not give rise to the vortical movement of the plume material. Instead, this zone is filled up by the nearest background molecules.

To determine the flow regions with potentially maximum $n_{\text {AlO }}^{0}$ production rate, we consider the calculated distribution of the product of the number densities of $\mathrm{Al}$ and $\mathrm{O}_{2}$, $n_{p}(r, Z)=n_{\mathrm{Al}} n_{\mathrm{O}}$ (Fig. 13). The $n_{p}$ distribution reveals a surprising similarity with the experiments, in spite of the fact that $n_{p}$ represents only one of the factors determining the production of $\mathrm{AlO}$. Most remarkably a spatial distribution with similar two maxima at the plume periphery can be observed at $t=6.3 \mu \mathrm{s}$. These maxima can be explained if we compare the calculated $n_{p}$ with the velocity flow field. The comparison indicates that one of the maxima, which is closer to the target, results from the backward particle fluxes. In fact, the backward fluxes, which are composed of both ablated atoms and gas molecules, have been observed in our calculations. The maximum of these backward flows is at the position of the first $n_{p}$ maximum. The second maximum results from the dynamics of the plume expansion and mixing with the gas. The ablated particles flying near the plume axis are stronger decelerated by the gas than those flying at oblique directions because of the preferential plume expansion in the direction of the outward target normal. At a certain time, the plume starts preferentially expanding in radial (oblique) directions. As a result, the plume-gas mixing is more intense at the plume periphery than near the plume axis.

The observed spatial distributions of the $n_{\mathrm{AlO}}^{0}$ can be, thus, explained by the dynamics of the plume-gas interaction and mixing, without appealing to such a complex phenomenon as the vortex. We emphasize, however, that the jet analogy may be more applicable under different experimental conditions, such as, a longer pulse time, a less-spherical plume, a denser plume and ambient gas, etc. Boundary conditions at the target can also influence the flow field. Under certain conditions, furthermore, ablated flows may turn to be turbulent, particularly at the zone of plume-gas mixing. The plume velocity required for this effect should be high enough to justify the criterion for the transition to turbulence, $\operatorname{Re}>10^{4}$, where $\operatorname{Re}=(U d \rho) / \mu$ is the Reynolds number, $U$ is the flow velocity, $d$ is the characteristic length, $\rho$ is the density, and $\mu$ is the viscosity. In ablated flows, Re may vary from several tens to thousands. In addition, during laser ablation, conditions can be realized for Rayleigh-Taylor-type instabilities at the plume-gas interface. Both of these effects (turbulence and instabilities) can, in principle, promote the mixing of the ablated and background species. These points are beyond the scope of the present paper.

\section{E. Plume expansion with diffuse reflection from the target}

To study the role of the boundary conditions at the irradiated target on the dynamics of laser plume evolution, several calculations are performed with diffuse reflection of the plume and gas species from the target. The obtained density distributions are shown in Fig. 14. One can observe the formation of a new density maximum in the vicinity of the target, in addition to the expanding plume. This second "plume" is composed of the ablated particles that are scattered back to the target and are then reflected. Because all the backscattered ablated species reenter the flow and interact, they form the new maximum near the target. In this case, like in the calculations with the particle absorption at the target, no vortices are obtained in the velocity flow fields. 


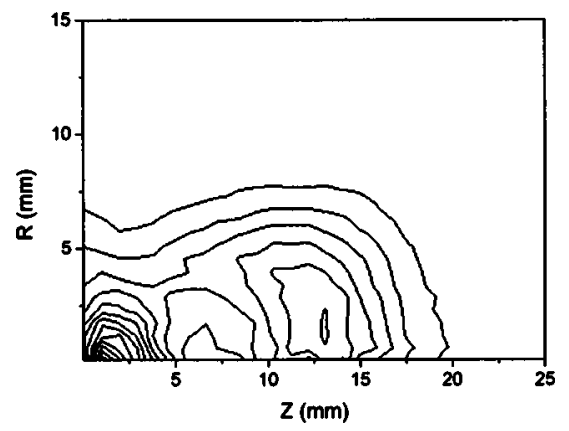

FIG. 14. Spatial distribution of the plume number density as a function of time obtained in calculation with diffuse reflection from the irradiated surface. Here, oxygen pressure $P_{b}=7 \mathrm{~Pa}$. Simulation results are obtained at $t=2.7 \mu \mathrm{s}$ from the beginning of the laser pulse.

Similar results, where plume particles formed another density maximum near the target, were previously observed in several experiments $[2,7,39,49]$. Unlike the first maximum, which streams away from the target, the second one was almost stationary. The observation of this stationary region was previously attributed to the collisions between the plume ejectants in the high-pressure Knudsen-layer region of the initial expansion with backward-moving material [7]. Our results support the role of the backscattering particles in these observations. We note, however, that typically the ejected species are scattered backward during longer time than the lifetime of the Knudsen layer. When ablation is performed in a background gas, the backscattering continues for several microseconds, whereas the Knudsen layer exists only during the laser pulse [50].

\section{SUMMARY AND CONCLUSIONS}

We have presented a combined continuous-microscopic model developed for the simulation of the expansion of laser-induced plasma plumes into background gases. The developed approach bridges the gap between the Monte Carlo modeling of the plume expansion in a low-pressure regime, and hydrodynamical models in the high-pressure regime. The proposed combined LP-DSMC model describes both the high-rate laser ablation and the collective motion of the laser-ablated species and the background-gas particles. The method is applicable for different background pressures, and is particularly suitable for the investigations of such effects as the mass diffusion and the energy exchange between the plume and the background gas, as well as the transition of the plume to the free flight regime and the interaction of the ablation products with solid surfaces.

The advantages of the model have allowed us to investigate the dynamics of the laser plume expansion for a wide range of background pressure. Three distinct regimes of the laser plume expansion have been unidentified. These regimes include (i) almost free expansion at small pressure (lower than $7 \mathrm{~Pa}$ for $\mathrm{Al}$ in $\mathrm{O}_{2}$ ), (ii) diffusion-driven expansion at moderate pressures (from 7 up to about $70 \mathrm{~Pa}$ in our case), and (iii) strong plume and gas compression (snowplough) at high pressure (higher than $70 \mathrm{~Pa}$ ). In the third case, the plume has been found to equilibrate through pulsations. The effect of the mixing of the plume species with backgroundgas molecules has been, furthermore, correlated with the observed pressure regimes. Thus, at small pressures, the gas species easily penetrate into the plume as soon as the plume density drops down due to the plume expansion. At moderate pressures, and when radial expansion sets in, the mixing is stronger at the plume periphery. At high pressures, the plume-gas mixing is complicated by the formation of the compressed layers in the plume and in the background gas. As a result, both the mass and energy exchange between the plume and the gas takes place only in the region between the gas and plume density maxima.

Calculation results have been used to investigate the snowplough effect. Based on the calculated density profiles for the plume and the background gas, we have determined the value of the background pressure, which is needed for the beginning of the plume snowplowing. For the ablation into $\mathrm{O}_{2}$, this pressure is found to be about $70 \mathrm{~Pa}$. The experimental results agree with the calculations.

The simulation results have been, furthermore, compared with the measured distributions of the ground-state number density of molecules produced by chemical reactions during reactive laser ablation. The density maps of the $\mathrm{AlO}$ formed during the laser ablation of aluminum in oxygen have been analyzed based on the experimental and modeling results. At high background pressure $(70 \mathrm{~Pa})$, the maximum of $n_{\mathrm{AlO}}^{0}$ has been found at the plume front near the axis, whereas two maxima have been observed at the plume periphery at lower pressure $(13 \mathrm{~Pa})$. The comparison of the experimental results with the calculated velocity flow field has suggested that these observations can be explained by the structure of the flow formed as a result of the plume-gas interaction. Thus, the maximum, which is closer to the target, results from the backscattered flows of the ablated and gas species. The second maximum, which is farther from the target, has been attributed to the radial plume expansion and plume-gas mixing at the oblique directions. Thus, our model has allowed to localize the molecular formation during reactive laser ablation experiments.

Despite a number of simplifications, the presented modeling has provided a physical picture of the complicated phenomenon of laser plume expansion into a background gas. This picture has yielded the explanations of a number of experimental results obtained in the presence of both inert and reactive background gases. The effects of the background gas on the ablated particles are extremely significant during the PLD of thin films. In fact, a careful choice of the filling atmosphere permits the high-rate deposition without damage of the films. In addition, thin film qualities, such as the crystalline or amorphous structure, are strongly affected by the energy of the species arriving at the substrate. The study is of interest for the interpretation of the reactive laser ablation experiments, in which plume-gas mixing is critical for the reactive molecule production. The developed approach can be useful to study the formation of nanoparticles during laser ablation. 
Finally, we note that we have considered the expansion of laser plasma. The proposed model can be, however, easily adapted for expanding plasmas obtained by a different method, as, for example, plasma torch and other techniques where strong density variations take place.

\section{ACKNOWLEDGMENT}

T.E.I. gratefully acknowledges financial support from the Centre National de la Recherche Scientifique (CNRS) of France.
[1] Pulsed Laser Deposition of Thin Films, edited by D. B. Chrisey and C. K. Hubler (Wiley, New York, 1994).

[2] Laser Ablation of Electronic Materials, Basic Mechanisms and Applications, edited by E. Fogarassy and S. Lazare (Elsevier Science, Amsterdam, 1992).

[3] W. Marine, B. Luk'yanchuk, and M. Sentis, Le Vide 2/4, 440 (1998).

[4] D. Dijkkamp, T. Venkatesan, X. D. Wu, S. A. Shaheen, N. Jisrawi, Y. H. Min-Lee, W. L. McLean, and M. Croft, Appl. Phys. Lett. 51, 619 (1987).

[5] E. Fogarassy, C. Fuchs, A. Slaoui, S. de Unamuno, J. P. Stoquert, W. Marine, and B. Lang, J. Appl. Phys. 76, 2612 (1994).

[6] W. Marine, M. Gerri, J. M. Scotto d'Aniello, M. Sentis, Ph. Delaporte, B. Forestier, and B. Fontaine, Appl. Surf. Sci. 54, 264 (1992).

[7] D. B. Geohegan, Appl. Phys. Lett. 60, 2732 (1992).

[8] D. B. Geohegan and D. N. Mashburn, Appl. Phys. Lett. 55, 2345 (1989).

[9] C. Vivien, J. Hermann, A. Perrone, C. Boulmer-Leborgne, and A. Luches, J. Phys. D 31, 1263 (1998).

[10] J. C. S. Kools, S. H. Brongersma, E. van de Riet, and J. Dieleman, Appl. Phys. B: Lasers Opt. B53, 125 (1991).

[11] F. Sakeek, T. Morrow, W. G. Graham, and D. G. Walmsley, Appl. Phys. Lett. 59, 3631 (1991).

[12] S. I. Anisimov, D. Bäuerly, and B. S. Luk'yanchuk, Phys. Rev. B 48, 12076 (1993).

[13] K. L. Saenger, J. Appl. Phys. 70, 5629 (1991).

[14] R. Kelly and R. W. Dreyfus, Nucl. Instrum. Methods Phys. Res. B 32, 314 (1988).

[15] R. Kelly, J. Chem. Phys. 92, 5047 (1990).

[16] I. NoorBatcha, R. R. Lucchese, and Y. Zeiri, Phys. Rev. B 36, 4978 (1987).

[17] D. Sibold and H. M. Urbassek, Phys. Rev. A 43, 6722 (1991).

[18] H. M. Urbassek and D. Sibold, Phys. Rev. Lett. 70, 1886 (1993).

[19] J. C. S. Kools, J. Appl. Phys. 74, 6401 (1993).

[20] L. V. Zhigilei and B. Garrison, Appl. Phys. Lett. 74, 1341 (1999).

[21] T. E. Itina, A. A. Katassonov, W. Marine, and M. Autric, J. Appl. Phys. 83, 6050 (1998).

[22] T. E. Itina, W. Marine, and M. Autric, J. Appl. Phys. 92, 3536 (1997).

[23] T. E. Itina, V. Tokarev, W. Marine, and M. Autric, J. Chem. Phys. 106, 8905 (1997).

[24] T. E. Itina, L. Patrone, W. Marine, and M. Autric, Appl. Phys. A: Mater. Sci. Process. 69, S59 (1999).

[25] F. Garrelie, C. Champeaux, and A. Catherinot, Appl. Phys. A:
Mater. Sci. Process. 69, S55 (1999).

[26] A. D. Akhsakhalyan, S. V. Gaponov, V. I. Luchin, and A. P. Chirimanov, Zh. Tekh. Fiz. 58, 1885 (1988) [Sov. Phys. Tech. Phys. 33, 1885 (1988)].

[27] A. V. Bulgakov and N. M. Bulgakova, J. Phys. D 28, 1710 (1995).

[28] A. V. Bulgakov and N. M. Bulgakova, J. Phys. D 31, 693 (1998).

[29] N. Arnold, J. Gruber, and J. Heitz, Appl. Phys. A: Mater. Sci. Process. 69, S87 (1999).

[30] A. V. Gusarov, A. G. Gnedovets, and I. Smurov, J. Appl. Phys. 88, 4352 (2000).

[31] J. N. Leboef, K. R. Chen, J. M. Donato, D. B. Geohegan, C. L. Liu, A. A. Puretzky, and R. F. Wood, Phys. Plasmas 3, 2203 (1996).

[32] J. N. Leboeuf, R. F. Wood, R. K. Chen, A. A. Puretzky, and D. B. Geohegan, Phys. Rev. Lett. 79, 1571 (1997).

[33] H. C. Le, D. E. Zeitoun, J. D. Parisse, M. Sentis, and W. Marine, Phys. Rev. E 62, 4152 (2000).

[34] Y. B. Zeldovich and Yu. P. Raizer, in Physics of Shock Waves and High-Temperature Hydrodynamic Phenomena, edited by W. D. Hayes and R. F. Probstein (Academic, London, 1966).

[35] L. I. Sedov, Similarity and Dimensional Methods in Mechanics (Academic, London, 1959).

[36] D. B. Geohegan, in Excimer Lasers, Vol. 256 of NATO Advanced Studies Institute, Series E: Applied Sciences, edited by L. D. Laude (Kluwer Academic, Dordrecht, 1994), p. 165.

[37] G. A. Bird, Molecular Gas Dynamics and the Direct Simulation of Gas Flows (Clarendon, Oxford, 1994).

[38] T. E. Itina, J. Appl. Phys. 89, 740 (2001).

[39] F. Garrelie, Ph.D. thesis, University of Limoges, France, 1998.

[40] T. E. Itina, J. Appl. Phys. 86, 4709 (1999).

[41] C. Dutouquet and J. Hermann, J. Phys. D 34, 3356 (2001).

[42] O. M. Belotserkovskii, J. Comput. Phys. 5, 587 (1970).

[43] T. E. Itina, W. Marine, and M. Autric, Appl. Surf. Sci. 710, 154 (2000); 710, 60 (2000).

[44] C. J. Knight, AIAA J. 17, 519 (1979).

[45] S. I. Anisimov, Zh. Eksp. Teor. Fiz. 54, 339 (1968) [Sov. Phys. JETP 27, 182 (1968)].

[46] A. Peterlongo, A. Miotello, and R. Kelly, Phys. Rev. E 50, 4716 (1994).

[47] G. A. Bird, Prog. Astronaut. Aeronaut. 51, 323 (1977).

[48] D. J. Lichtenwalner, O. Auciello, R. Dat, and A. I. Kingon, J. Appl. Phys. 74, 7497 (1993).

[49] R. Kelly, A. Miotello, A. Mele, and A. G. Guidoni, Exp. Methods Phys. Sci. 30, 251 (1998).

[50] T. E. Itina, W. Marine, and M. Autric, J. Appl. Phys. 85, 7905 (1999). 